\title{
Investigations in the amplitude of sounded piano tones
}

Caroline Palmer, and Judith C. Brown

Citation: The Journal of the Acoustical Society of America 90, 60 (1991); doi: 10.1121/1.401249

View online: https://doi.org/10.1121/1.401249

View Table of Contents: https://asa.scitation.org/toc/jas/90/1

Published by the Acoustical Society of America

\section{ARTICLES YOU MAY BE INTERESTED IN}

Numerical simulations of piano strings. I. A physical model for a struck string using finite difference methods The Journal of the Acoustical Society of America 95, 1112 (1994); https://doi.org/10.1121/1.408459

Generation of longitudinal vibrations in piano strings: From physics to sound synthesis

The Journal of the Acoustical Society of America 117, 2268 (2005); https://doi.org/10.1121/1.1868212

Some empirical observations on sound level properties of recorded piano tones

The Journal of the Acoustical Society of America 93, 1136 (1993); https://doi.org/10.1121/1.405561

Modeling and simulation of a grand piano

The Journal of the Acoustical Society of America 134, 648 (2013); https://doi.org/10.1121/1.4809649

False beats in coupled piano string unisons

The Journal of the Acoustical Society of America 115, 885 (2004); https://doi.org/10.1121/1.1636461

Measurement and reproduction accuracy of computer-controlled grand pianos

The Journal of the Acoustical Society of America 114, 2273 (2003); https://doi.org/10.1121/1.1605387 


\title{
Investigations in the amplitude of sounded piano tones
}

\author{
Caroline Palmer \\ Psychology Department, Ohio State University, 1885 Neil Avenue, Columbus, Ohio 43210 \\ Judith C. Brown \\ Massachusetts Institute of Technology Media Laboratory, Cambridge, Massachusetts 02319 \\ and Physics Department, Wellesley College, Wellesley, Massachusetts 02181
}

(Received 8 November 1990; accepted for publication 27 March 1991)

The relationship between final hammer velocity and maximum amplitude of radiated piano sound was investigated. Piano tones with varying hammer velocities were produced by a computer-monitored acoustic piano containing optical sensors and solenoids, and the sounded tones were recorded and digitized for analysis. Maximum amplitudes over the duration of the sounded tones were linearly proportional to piano hammer velocities for a range of frequencies and hammer velocities. Changes in room acoustics did not alter the linear relationship.

Measurements of maximum amplitudes of individual tones and combined tones (dyads) also indicated a linear relationship between the sum of the maximum amplitudes of the individual tones and the maximum amplitude of the dyads. These findings indicate that the principle of superposition holds for peak amplitudes of sounded piano tones. Findings are discussed with regard to production and perception of musical dynamics.

PACS numbers: 43.75.Mn

\section{INTRODUCTION}

The relationship between production of musical dynamics in piano performance and its subsequent perception is largely unstudied, with a few exceptions (Fletcher et al., 1962; Nakamura, 1987). Musical dynamics refer to changes in perceived loudness, which arise from an interplay of intensity, timbral, and temporal changes afforded by a particular musical instrument (Handel, 1989; Lerdahl and Jackendoff, 1983). The piano, a musical instrument with a large range of intensity and temporal change but a relatively fixed timbral range, provides an excellent domain for study of the mechanisms leading to changes in musical dynamics. Preliminary to an understanding of the musicians' use of dynamics, we need to examine the relationship between radiated piano sound and hammer velocities, the pianist's primary means of controlling loudness. We describe here investigations of the influence of hammer velocity on the amplitude of sounded piano tones.

Factors characterizing the mechanisms of production of piano sound have been well documented (Weinreich, 1977; Hall, 1987a; Hall and Clark, 1987; Askenfelt and Jansson, 1988; Boutillon, 1988; Hall and Askenfelt, 1988). Sound generation in the piano depends on many factors, most important of which are the motion of the strings caused by the impact of hammers and the impedance radiation characteristics of the soundboard. When a piano key is depressed, the piano action transmits energy from the pianist's fingers to the appropriate hammers and strings. A hammer is thrown against the strings, setting them into vibration. String vibrations are then transmitted via the bridge to the soundboard, the main sources of radiated sound (Boutillon, 1988; Hall, 1990; Rossing, 1990).

Several studies of piano string motion suggest that amplitudes of string vibrations are proportional to the final hammer velocity (Askenfelt and Jansson, 1988; see also
Hall's model of string vibration amplitudes, 1987a ). Careful measurements of the amplitude of initial string pulses revealed a linearity with final hammer velocity (Askenfelt and Jansson, 1988), suggesting that, if soundboard displacement is proportional to string motion, then the amplitude of radiated sound would be proportional to hammer velocity as well. Although recent studies suggest that the linear theory cannot account for spectral changes seen in the upper partials of string motion (Hall and Askenfelt, 1988; Hall, 1990), the nonlinear compliance of piano hammers may not substantially affect that part of the spectrum which dominates maximum amplitude. The experiments described below address the question of linearity between (final) hammer velocities and the maximum (or peak) amplitude of radiated sound.

Relative amplitude changes during the attach portion of musical sounds may be the most important perceptual cue to onset time and timbral identification, as well as to loudness. Perceptual experiments indicate that listeners are most sensitive to the initial portion of a sounded tone in their perception of timbre (Grey, 1977) and relative onset times (Rasch, 1978; Vos and Rasch, 1981). For most acoustic musical instruments, the amplitude changes over the duration of the radiated sound, with the greatest change in the attack, or time from zero amplitude to peak amplitude (Apel, 1973). In piano sound, the attack contains the most change in spectral energy, with all contributing harmonics decaying rapidly after peak amplitude. The role of the peak amplitude during the initial portion of a sound is important in perception of dynamic emphasis in both music and speech (Sundberg et al., 1983; Carlson et al., 1989), suggesting commonalities in auditory perception underlying the two domains.

We describe several experiments that address the relationship between amplitude of radiated piano sound and hammer velocity, the pianist's main method of controlling dynamic level. This relationship was studied with the aid of a 
computer-monitored acoustic grand piano, whose solenoids allowed precise control and generation of piano tones with varying frequencies and hammer velocities. We present evidence consistent with Hall's (1987a) model of a linear relationship between string vibration amplitudes and hammer velocity, suggesting that the maximum or peak amplitude of the radiated piano sound is proportional to the hammer velocity as well.

\section{EQUIPMENT}

A 9-ft 6-in. Bosendorfer SE computer-monitored concert grand piano, containing optical sensors and solenoids, allowed precise recording and playback of hammer velocities. Infrared photodetectors in the piano, placed near the position of impact of each hammer head on a string, measure the final velocity with which the hammer approaches the string. Solenoids calibrated for each key allowed precise reproduction of each hammer velocity. The final hammer velocity and keypress onset and offset times for each note event were controlled by an IBM/PC computer.

The computer-monitored piano sat in a rectangular room, approximately $9.5 \times 3.7 \times 3.7 \mathrm{~m}$ in length, width, and height, with painted concrete walls, a tiled floor, and suspended acoustic tile ceiling consisting of large pipes and other irregular geometrical shapes. The room was furnished with a computer workstation and electronics rack in addition to the grand piano and recording equipment used in the experiment. The reverberation time of the room was measured by recording and digitizing a handclap in the room and analyzing its decay after the completion of the experiments. Based on this measurement, we estimated a reverberation time of $0.3 \mathrm{~s}$ for the conditions of the first experiment (Hall, 1987b).

During the recording sessions, the piano lid was open and the music stand was down; no attempts were made to muffle the radiated sound with acoustic material. Recordings of the radiated sound were made with a Neumann KM84 cardioid (directional) microphone placed near a pianist's head position, centered over the middle of the piano keyboard approximately $0.5 \mathrm{~m}$ from, and directed toward, the soundboard edge. Thus the measurements of peak amplitude were made at one position in the nearfield under normal room conditions. The microphone was connected to a Sony MX-P21 mixer, which passed the signal to a 16-bit Sony digital audio tape recorder sampling at $48 \mathrm{kHz}$. The sound was transferred in analog form to computer and redigitized. To reduce the required computer space, analyses were compared at sampling rates of 48 and $32 \mathrm{kHz}$. Spectral analyses revealed little to no power in the frequency range over 16 $\mathrm{kHz}$; because there was no loss in information, analyses were conducted on the information sampled at $32 \mathrm{kHz}$.

Piano hammer velocities in the range of 0.25 to $4 \mathrm{~m} / \mathrm{s}$ were chosen to represent musical dynamic markings from pianissimo to forte (an approximate difference of $25 \mathrm{~dB}$ across the range of 45-70 dB sound-pressure level). The range of hammer velocities was based on common values recorded in performances on this piano (over $90 \%$ of hammer velocities from a set of piano performances of Western classical excerpts fell within this range; Palmer, 1988). In addition, the frequency range was chosen over 7 octaves (a frequency difference of approximately $2000 \mathrm{~Hz}$ across the range of 33-2096 Hz), allowing study of sounded amplitude across a wide range of hammer velocities and frequencies. $\mathbf{A}$ set of measurements of $\mathrm{C} 4$ (middle $C$ ) sounded at $1-\mathrm{m} / \mathrm{s}$ hammer velocity were recorded at the beginning of each experiment, to allow comparisons across recording sessions (because the gain setting of the recording equipment was readjusted at the beginning of each experiment).

The piano was calibrated before each recording for seven different hammer velocities at each frequency. The reliability of the recording equipment was determined by examining the standard error of repeated measurements for sounds produced with the same hammer velocity and frequency at the beginning and end of each recording session. The consistency of the repeated measurements, represented by the standard error, indicated less than $5 \%$ difference for the set of hammer velocities and frequencies being measured.

\section{MEASUREMENTS AND RESULTS}

\section{A. Peak amplitude proportional to hammer velocity}

The first experiment investigated the relationship of final hammer velocity to peak amplitude in the radiated piano sound. Two variables were altered: frequency and final hammer velocity. Seven frequencies (using C's ranging from $\mathrm{Cl}$ to $\mathrm{C}$, where $\mathrm{C4}=$ middle $\mathrm{C}$ ) were paired with five hammer velocities, including $0.25,0.5,1,2$, and $4 \mathrm{~m} / \mathrm{s}$. Ten repetitions of each combination of velocity and frequency were generated by computer, resulting in 350 measurements. Each tone was sounded for $500 \mathrm{~ms}$, followed by $1500 \mathrm{~ms}$ of silence. This was followed by the sounding of the next repetition. After the radiated sound was recorded and transferred to computer, numerical analyses were produced. The amplitude values were then scaled to a common range for all experiments reported, using the $\mathrm{C} 4$ calibration measurements recorded at the beginning of each session. Note that the absolute slope values are arbitrary both before and after the scaling, but the percentage difference in slopes remains the same. Comparisons will only be drawn between slopes within experiments.

Figure 1 contains the first $240 \mathrm{~ms}$ of sound for two tones, C3 sounded at the hammer velocities of $2 \mathrm{~m} / \mathrm{s}$ (mezzoforte) and $4 \mathrm{~m} / \mathrm{s}$ (forte). The peak amplitude, defined as the maximum of the absolute values of amplitude over the duration of each tone, is approximately doubled with the doubling of the hammer velocity. The position of peak amplitudes was stable across trials; it tended to shift less than three periods between repeated measurements of the same frequency.

The peak amplitude of each tone was determined by computer, by choosing the largest absolute value of the samples over the 500-ms duration of each tone. Figure 2 shows the mean peak amplitude across ten measurements for each combination of hammer velocity and frequency. Note that the peak amplitude increases linearly with hammer velocity with the exception of the smallest hammer velocity of 0.25 $\mathrm{m} / \mathrm{s}$, which yielded amplitudes similar to those of $0.5 \mathrm{~m} / \mathrm{s}$. To test the linear relationship between peak amplitude and hammer velocity, a linear regression model was fit, predict- 



FIG. 1. Sample waveforms from sounded tones: Beginning section (240 $\mathrm{ms}$ ) of tone $\mathrm{C} 3$ sounded at 2 - and $4-\mathrm{m} / \mathrm{s}$ hammer velocity. Peak amplitude marked with arrow (see text).

ing the peak amplitude from the hammer velocities, omitting $0.25 \mathrm{~m} / \mathrm{s}$. The linear regression model provided an excellent fit for each frequency, shown in Fig. 2, with a multiple regression coefficient greater than $0.99(p<0.001)$ for each frequency.

Figure 2 also indicates that the slopes associated with each fit are not ordered in terms of frequency; that is, the proportionality does not appear to be affected by the frequency range. To test for differences among slopes, 95\% confidence intervals were calculated for each slope. The only slope that significantly differed from the others (by $40 \%$ )

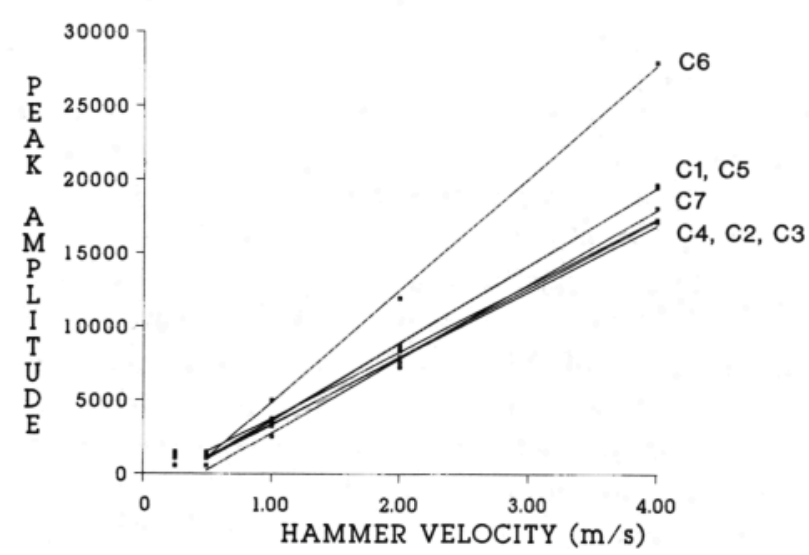

FIG. 2. Peak amplitude as a function of hammer velocity for seven frequencies with fitted linear regression. that of C6, as seen in Fig. 2. The variability of the measurements made at this frequency was much higher than for other frequencies, indicating that the range of hammer velocities produced by this solenoid was more variable than those of the other solenoids. With the exception of $\mathrm{C6}$, frequency did not significantly alter the proportionality between peak amplitude and hammer velocity, and the greatest difference between slope values was less than $15 \%$.

Although peak amplitude was highly correlated with final hammer velocity, other sound characteristics such as intensity integrated over some portion of the tone may be more closely related to musical dynamic level. To investigate this hypothesis, final hammer velocity was compared with other characteristics of the radiated sound. Simple correlations were computed between: hammer velocity and the integral of the absolute value of amplitude from the beginning of sound until time of peak amplitude (within $50 \mathrm{~ms}$ for all but the lowest frequency and hammer velocity combinations); hammer velocity squared and the integral of the intensity (amplitude squared) up to time of peak amplitude; and the same measurements for the entire duration of the tones. None of these variables showed as large a correlation with hammer velocity (or hammer velocity squared) as did peak amplitude.

Thus peak amplitude of radiated piano sound was linearly proportional to hammer velocity for a given frequency in these measurements. One possible limitation of the experimental procedure concerned the room acoustics; because the walls and floor were reflectant, the reverberant conditions may have affected the linear relationship. The next study attempted to replicate the linearity finding under different room acoustic conditions.

\section{B. Change in acoustic environment}

To extend the previous findings, changes were made in room acoustics and in the range of frequencies measured. The same room used in the previous study was now equipped with heavy curtains covering $2 / 3$ of each wall (from ceiling to floor) to decrease the reverberation time. A second piano had also been added to the room, further decreasing the volume-to-surface ratio. The reverberation time for this acoustic environment, based on measurements of a handclap (recorded and digitized under these conditions) was estimated to be approximately half of its previous value of $0.3 \mathrm{~s}$.

Instead of the $\mathbf{C}$ frequencies, $\mathbf{F}$-sharps were measured from octaves 1-7. The stimulus durations and hammer velocities were the same as in the previous measurements, except that the $0.25-\mathrm{m} / \mathrm{s}$ hammer velocity was not included (because the previous measurements had shown no difference from the $0.5-\mathrm{m} / \mathrm{s}$ hammer velocity), and a hammer velocity of $3 \mathrm{~m} / \mathrm{s}$ was included. Five repetitions of each hammer velocity and frequency were recorded, because the variability of the previous measurements was low.

Figure 3 shows the peak amplitude as a function of hammer velocity, for each frequency. Again, peak amplitude is linearly proportional to hammer velocity. A multiple regression model was fit for each frequency, predicting the peak amplitude from the hammer velocity for the 25 measurements. The lines in Fig. 3 demonstrate the fits for each fre- 


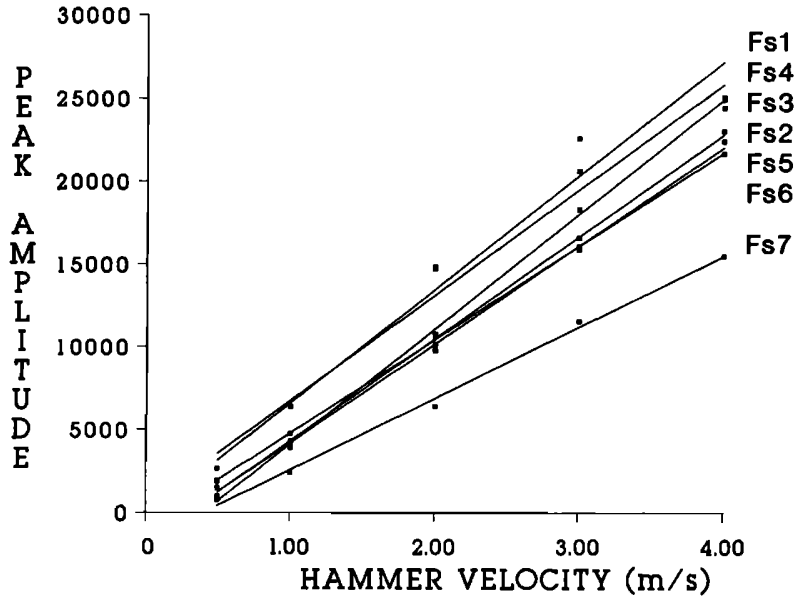

FIG. 3. Peak amplitude as a function of hammer velocity for seven frequencies under altered room acoustic conditions, with fitted linear regression.

quency. The linear regression model provided an excellent fit, with a multiple correlation coefficient $>0.99(p<0.001)$ for each frequency.

Figure 3 also indicates that the linear relationship was not affected by frequency range, because the slopes associated with each line are not ordered in terms of frequency. To test for any differences among slopes, $99 \%$ confidence intervals were again calculated for each slope. Again, the slopes did not differ significantly (the largest difference in slopes was $18 \%$ ), with the exception of Fs7, whose slope is lower than that of the other frequencies. Thus the range of differences among slopes was similar to that of the first experiment, and the absolute differences in measurements are attributable to differences in gain settings during the recording sessions (the range of measurements from the first setting was approximately half that of the second setting, and the absolute difference in slopes in Fig. 2 was approximately half that of the slope differences in Fig. 3).

Peak amplitude of radiated piano sound was again linearly proportional to hammer velocity for a given frequency. These results replicate those of the previous experiment, with changes in room acoustics and frequencies. It is not clear, however, that multiple (simultaneously sounded) tones would hold the same relationship between peak amplitude and hammer velocity. The next study was designed to test this hypothesis.

\section{Principle of superposition}

The peak amplitude of sounded dyads (two simultaneously sounded tones) were compared with the peak amplitudes of their individual component tones in the next study. The question addressed was whether the principle of superposition holds for peak amplitudes; is the peak amplitude of dyads proportional to the sum of the peak amplitudes of the individual tones? This would imply that the response of the soundboard is linear; its vibration in response to a dyad is the sum of the vibrations produced by the individual tones sounded separately. To address this question, radiated sound from common musical dyads and their individual component tones was recorded.
The previous experiments employed frequencies with simple integer ratios (2:1 or an octave apart). In this study, frequencies were chosen that differed in ratio. The common musical intervals of the minor third and the fifth were included, along with the tritone as an example of an interval with few overlapping harmonics. These three intervals were chosen to represent a range of frequency ratios between the individual tones: fifths with the most common harmonics (3:2 ratio) of these three intervals, minor thirds with next most common harmonics (6:5 ratio), and the tritone with the fewest common harmonics. If frequency ratios affect the peak amplitudes of the combined tones, then the relationship between peak amplitude and hammer velocity may differ for each interval.

Each dyad contained C4 (middle $\mathrm{C}$ ) as its base, played at a hammer velocity of $1 \mathrm{~m} / \mathrm{s}$ (a dynamic level of mezzoforte). Thus the three dyads in this study were: C4/Ef4, $\mathrm{C} 4 / \mathrm{Fs} 4$, and C4/G4. Hammer velocities for the Ef4, Fs4, and G4 were the same as in the first experiment: These were $0.5,1,2$, and $4 \mathrm{~m} / \mathrm{s}$. Ten recordings were made for each individual tone and for each of the three dyads at each of the four hammer velocities.

First, the relationship between peak amplitude and hammer velocity for the individual tones was examined. Figure 4 depicts the peak amplitude as a function of hammer velocity, for the three individual tones (Ef4, Fs4, and G4). Each of the three tones was fit by a linear regression analysis, predicting the peak amplitude from hammer velocity; the lines in Fig. 4 indicate those fits. The linear relationship seen previously was replicated, with a significant fit (multiple regression coefficient $=0.99, p<0.001$ ) for each frequency.

Next, the superposition of the individual tones in the dyads was examined. The dyads' peak amplitudes were compared with the peak amplitude of the individually sounded tones. For each dyad, the sum of the peak amplitudes of its individual component tones was calculated. Figure 5 displays the mean peak amplitude for the dyads as a function of the sum of the mean peak amplitudes for the individual tones. The linear relationship was fit with a regression analysis, and the regression fits for each dyad are shown in Fig. 5.

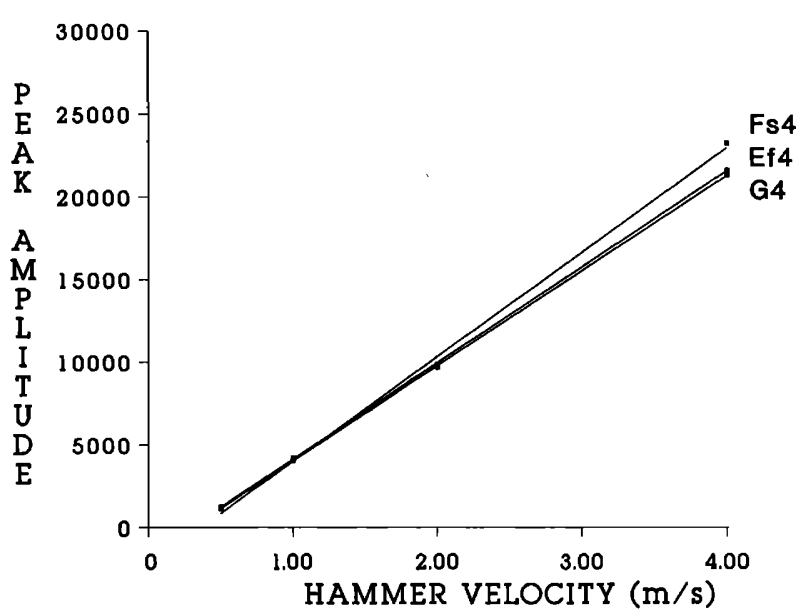

FIG. 4. Peak amplitude as a function of hammer velocity for three individual tones with fitted linear regression. 


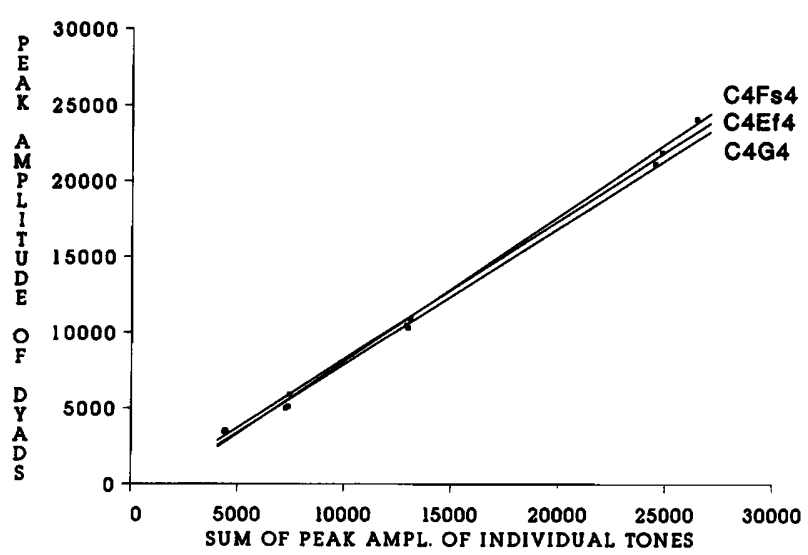

FIG. 5. Peak amplitude of dyads as a function of summed peak amplitudes of individual tones for three frequency combinations, with fitted linear regression.

The multiple regression coefficient is greater than 0.99 in each fit $(p<0.01)$ and the slopes ranged from 0.90 to 0.95 , supporting the principle of superposition. The intercepts were small and negative; the deviations of the intercepts from zero and slopes from 1 are due in part to differences in phase of the two superposed waveforms. The observed additivity is primarily due to the fact that the peak amplitudes for each waveform occurred at approximately the same position
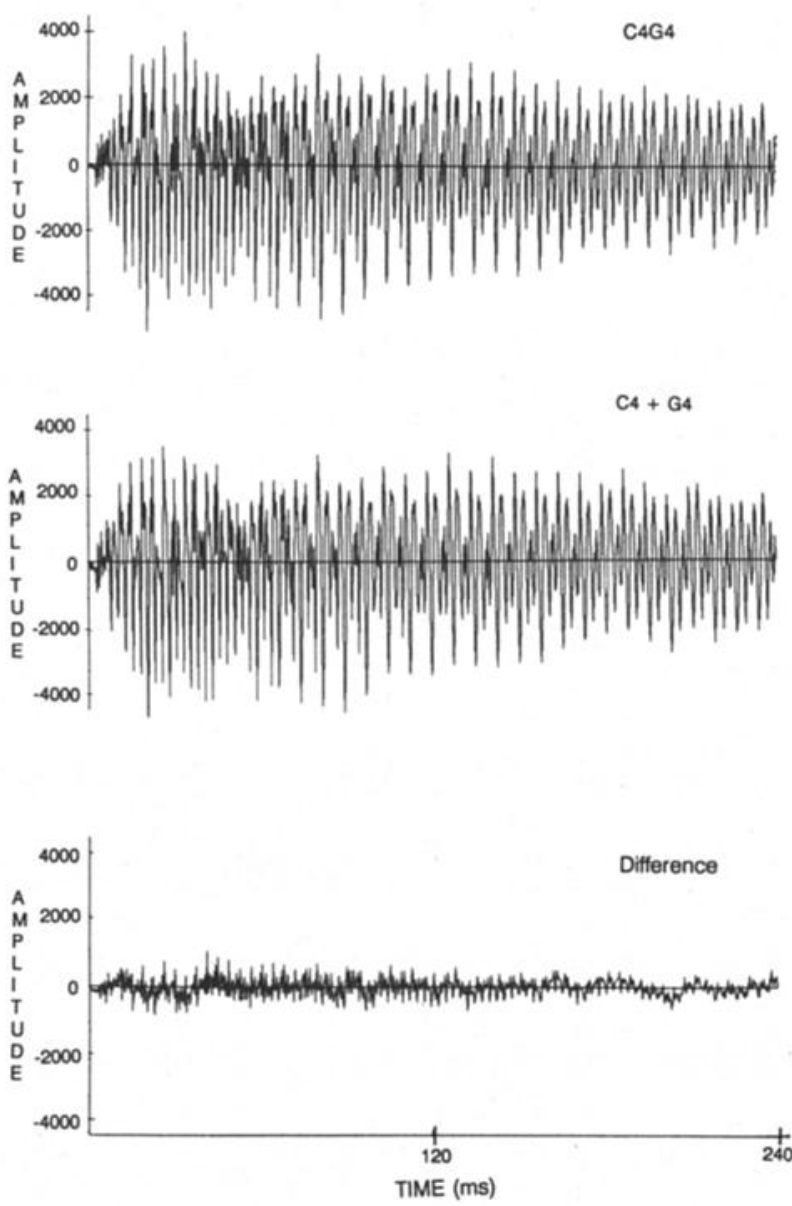

FIG. 6. Waveforms for sounded dyad C4G4 at hammer velocity of $1 \mathrm{~m} / \mathrm{s}$ (top), sum of individual tones $\mathrm{C} 4$ and $\mathrm{G} 4$ at $1 \mathrm{~m} / \mathrm{s}$ (middle), and their difference (sum of individual tones minus dyad) (bottom). (within three periods), and amplitudes over several surrounding cycles were approximately equal to the peak amplitude, as shown in the example given below.

Figure 6 demonstrates both the sounded dyad (top) and the superposition (middle) of the individual tones of $\mathrm{C} 4$ and G4 at $1-\mathrm{m} / \mathrm{s}$ hammer velocity, summed sample by sample. This example represents an extreme case in which the peak amplitudes of the individual tones were far apart in time. The relative phase of the individual tones in the computed superposition was determined by shifting the waveforms of the individual tones by three samples with respect to each other (a phase shift of approximately $0.1 \mathrm{~ms}$, which is less than the 1.25-ms temporal resolution of the hammer movements). Because the waveforms do not vary much in amplitude from the maximum over the critical time range, the peak amplitude for the sum of the individual tones is still proportional to the peak amplitude of the dyads (Fig. 5).

To measure how closely the principle of superposition was obeyed for the entire waveform, the difference between the computed sum of the individual waveforms and the dyad was calculated. The waveform phases were matched by shifting the dyad 5 samples (less than $0.16 \mathrm{~ms}$ ) with respect to the computed sum. The dyad was then subtracted, sample by sample, from the sum of the individual tones. Figure 6 (bottom) depicts the difference (sum - dyad) between the two waveforms. The small difference indicates that the principle of superposition is obeyed with very small deviations. This is consistent with the findings of Fig. 5, showing small deviations in peak amplitudes of dyads predicted from the summed peak amplitudes of individual tones.

\section{Superposition in a different frequency range}

Previous measurements of string-hammer interaction indicate that string-hammer contact duration changes with frequency and hammer velocity (Askenfelt and Jansson, 1988; Hall and Askenfelt, 1988), and the energy present in the upper harmonics increases with hammer velocity. The question of how these changes affect maximum amplitude was addressed by studying the principle of superposition in a different frequency and hammer velocity range. Measurements were made of the same common musical intervals: the minor third, the fifth, and the tritone. Individual tones were chosen from 2 octaves (Ef3, Fs3, G3, Ef5, Fs5, G5), and dyads were created by pairing each individual tone with the C in its octave (Ef3 was paired with C3; Ef5 was paired with C5). Hammer velocities were $0.5,1,1.5,2,3$, and $4 \mathrm{~m} / \mathrm{s}$; each individual tone and dyad was paired with each hammer velocity to create 72 combinations. $\mathrm{C} 3$ and C5 were always presented at $1 \mathrm{~m} / \mathrm{s}$ (a dynamic level of mezzoforte). Ten recordings were made for each sound.

A linear regression analysis tested whether the peak amplitude of the dyads was proportional to the sum of the peak amplitudes of the individual tones. Figure 7 displays the fits, which were good (multiple regression coefficients $>0.99$, $p<0.001$ ). The peak amplitude of dyads is proportional to the sum of the peak amplitudes of the individual tones despite the changes in frequency and hammer velocities. With one exception (the fit for C5G5), the slopes did not differ (ranging from $0.81-0.96$ ) and the intercepts were again 




FIG. 7. Peak amplitude of dyads as a function of sum of peak amplitudes of individual tones for extended frequency range, with fitted linear regression.

small and negative. Some of the data contributing to the C5G5 slope were inadvertently erased from the computer, and the data had to be recovered from tape and redigitized. This error may account for the notable difference in the C5G5 slope; however, the same linear relationship between the sum of peak amplitudes of individual tones and the peak amplitude of the dyads still held.

To examine further the effects of frequency range and hammer velocity on upper harmonics, a spectral analysis was conducted on the waveforms. Results of the Fourier transform for a sample dyad (C5Fs5) are shown in Fig. 8, and indicate rapid decay in the amplitudes of lower harmonics, and little to no energy in the upper harmonics. Thus the proportionality of peak amplitudes remained unaffected by the small changes in relative contributions of upper harmonics.

\section{DISCUSSION}

First, these studies indicate a linear relationship between the peak amplitude of individual tones and the hammer velocities that produced them. This finding implies that the series of events in piano mechanisms of sound produc-

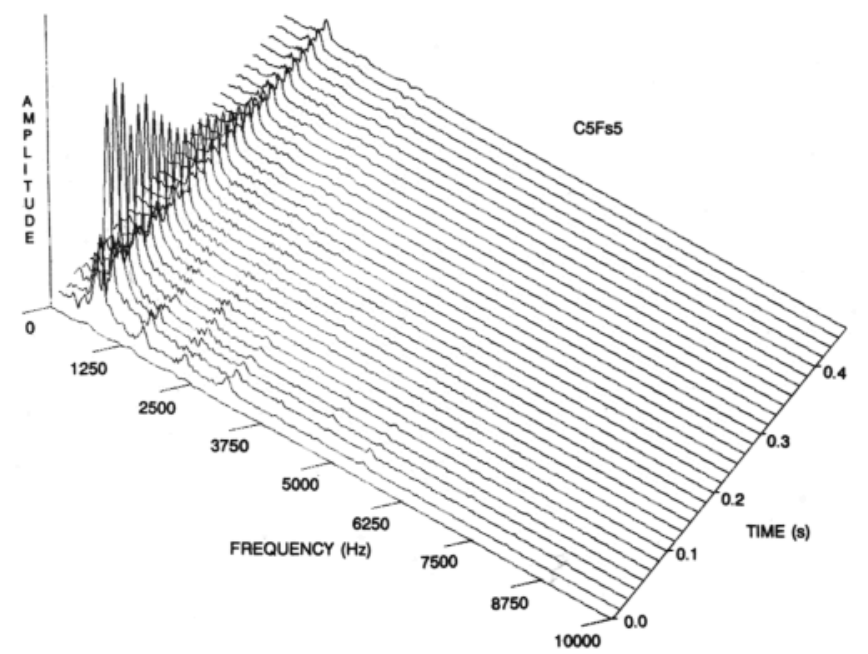

FIG. 8. Graphical depiction of spectral analysis of dyad C5Fs5 waveform at 4-m/s hammer velocity. tion, from hammers, to strings, to bridge, and to soundboard, approximates linearity for peak amplitudes in the range studied here. This finding is at first surprising because the radiated sound reflects the sum of the normal modes of vibration of both strings and soundboard and implies a linear coupling for those modes contributing to peak amplitude measurements. However, we have examined a set of hammer velocities for a given frequency, and although the hammer's velocity was varied, exactly the same modes of a particular string were excited. In addition, because string resonances with periods shorter than the contact duration of hammer with string are only weakly excited (Hall, 1990), the frequencies used here generated little energy in the upper harmonics for each sounded event, and peak amplitude was primarily determined by the first few spectral components. Thus the linear relationship between peak amplitude and hammer velocity may be more predictable than it would first appear.

The spectral analyses described above indicated that the energy present at higher harmonics dropped off quickly in time and the peak amplitude of radiated sound was composed primarily of the fundamental and a smaller contribution of the lower harmonics. Thus, although changes in hammer velocity and hammer-string contact duration alter the relative contributions of upper harmonics to the sounded tone (Hall and Askenfelt, 1988), they may not substantially alter the peak amplitude. Whereas a linear theory cannot account for the rapid decrease in amplitude of higher string modes, the small magnitudes of these spectral components had little effect on the peak amplitudes observed here.

Second, these studies indicate that the principle of superposition holds for peak amplitudes of dyads obtained from the peak amplitudes of two individual tones. This result held true over a range of frequency and hammer velocity combinations. Together with the linearity seen between hammer velocity and peak amplitude for individual tones, this finding is encouraging for the study of perceived dynamics in piano performance, because it suggests a functional mapping of individual hammer velocities to peak amplitudes of sounded chords. Hammer velocity is one of the few parameters under a pianist's direct control; therefore, it is important that combinations of hammer velocities can be mapped to amplitude characteristics of radiated sound that are crucial for listeners. Future work may extend the linearity and superposition findings to a more natural musical setting and a perceptual domain. These findings may aid investigation of the relationship between the pianist's use of force and timing and the musical dynamics perceived by the listener.

\section{ACKNOWLEDGMENTS}

This work was supported in part by an Ohio State University Small Grant and Grant 1R29-MH45764 from NIMH to the first author and by a Marilyn Brachman Hoffman Fellowship to the second author. We thank Donald Hall, William Strong, and an anonymous reviewer for comments on an earlier draft and the members of the Music and Cognition Group in the MIT Media Lab, where some of this research was conducted. 
Apel, W. (1973). Harvard Dictionary of Music (Harvard U.P., Cambridge, MA).

Askenfelt, A., and Jansson, E. (1988). "From touch to string vibrationsthe initial course of the piano tone" (Royal Institute of Technology, Stockholm), Speech Trans. Lab. Q. Prog. Stat. Rep. 1, 31-109.

Boutillon, X. (1988). "Model for piano hammers: Experimental determination and digital simulation," J. Acoust. Soc. Am. 83, 746-754.

Carlson, R., Friberg, A., Fryden, L., Granstrom, B., and Sundberg, J. (1989). "Speech and music performance: parallels and contrasts," Cont. Mus. Rev. 4, 389-402.

Fletcher, H., Blackham, E. D., and Stratton, R. (1962). "Quality of piano tones," J. Acoust. Soc. Am. 34, 749-761.

Grey, J. M. (1977). "Multidimensional perceptual scaling of musical timbres," J. Acoust. Soc. Am. 61, 1270-1277.

Hall, D. E. (1980). Musical Acoustics: An Introduction (Wadsworth, Belmont, CA).

Hall, D. E. (1987a). "Piano string excitation II: General solution for a hard narrow hammer," J. Acoust. Soc. Am. 81, 535-546.

Hall, D. E. (1987b). Basic Acoustics (Harper \& Row, New York).

Hall, D. E. (1990). "The hammer and the string," in Five Lectures on the Acoustics of the Piano, edited by A. Askenfelt (R. Swedish Academy of Music, Stockholm), pp. 59-72.
Hall, D. E., and Askenfelt, A. (1988). "Piano string excitation V: Spectra for real hammers and strings," J. Acoust. Soc. Am. 83, 1627-1638.

Hall, D. E., and Clark, P. (1987). "Piano string excitation IV: The question of missing modes," J. Acoust. Soc. Am. 82, 1913-1918.

Handel, S. (1989). Listening (MIT, Cambridge, MA).

Lerdahl, F., and Jackendoff, R. (1983). A Generative Theory of Tonal Music (MIT, Cambridge, MA).

Nakamura, T. (1987), "The communication of dynamics between musicians and listeners through musical performance," Percept. Psychophys. 41, 525-533.

Palmer, C. (1988). "Timing in skilled music performance," unpublished doctoral dissertation, Cornell University.

Rasch, R. A. (1978). "The perception of simultaneous notes such as in polyphonic music," Acustica 40, 22-33.

Rossing, T. D. (1990). The Science of Sound (Addison-Wesley, Reading, MA).

Sundberg, J., Askenfelt, A., and Fryden, L. (1983). "Musical performance: A synthesis-by-rule approach," Comp. Mus. J. 7, 37-43.

Vos, J., and Rasch, R. (1981). "The perceptual onset of musical tones," Percept. Psychophys. 29, 323-335.

Weinreich, G. (1977). "Coupled piano strings," J. Acoust. Soc. Am. 62, 1474-1484. 